\title{
A variant of compressed sensing
}

\section{Basarab Matei and Yves Meyer}

\begin{abstract}
The famous Nyquist-Shannon sampling theorem has been recently improved by A. Olevskii and A. Ulanovskii. The present contribution is aimed at bridging the gap between their advance on irregular sampling and what is named "compressed sensing" in signal processing.
\end{abstract}

\section{Introduction}

This paper is motivated by some recent advances on what is now called "compressed sensing". Let us begin with a theorem by Terence Tao [8]. Let $p$ be a prime number and $\mathbb{F}_{p}$ be the finite field with $p$ elements. We denote by $\# E$ the cardinality of $E \subset \mathbb{F}_{p}$. The Fourier transform of $f \in l^{2}\left(\mathbb{F}_{p}\right)$ is denoted by $\hat{f}$. Let $M_{q}$ be the collection of all $f \in l^{2}\left(\mathbb{F}_{p}\right)$ such that the cardinality of the support of $f$ does not exceed $q$. Then Terence Tao proved that for $q<p / 2$ and for any set $\Omega$ of frequencies such that $\# \Omega \geq 2 q$, the mapping $\Phi: M_{q} \mapsto l^{2}(\Omega)$ defined by $f \mapsto \mathbf{1}_{\Omega} \hat{f}$ is injective. Here and in what follows, $\mathbf{1}_{E}$ will denote the indicator function of the set $E$. Tao's theorem is no longer true if $\mathbb{F}_{p}$ is replaced by $\mathbb{Z} / N \mathbb{Z}$ and if $N$ is not a prime.

We want to generalize this fact to functions $F$ defined on the unit square with applications to image processing. In a forthcoming work the hypothesis that $F$ is supported by the unit square will be removed. Here and in what follows the action takes place on $[0,1]^{2}$ identified to $(\mathbb{R} / \mathbb{Z})^{2}$. Since the unit square $[0,1]^{2}$ has been identified to $(\mathbb{R} / \mathbb{Z})^{2}$, the Fourier transform of $F \in$ $L^{1}\left([0,1]^{2}\right)$ is the sequence of its Fourier coefficients defined by

$$
\hat{F}(k)=\int_{0}^{1} \int_{0}^{1} \exp (-2 \pi i k \cdot x) F(x) d x, \quad k \in \mathbb{Z}^{2} .
$$

2000 Mathematics Subject Classification: Primary: 42B10; Secondary: 42C30.

Keywords: Fourier expansions, compressed sensing, irregular sampling. 
To generalize Tao's theorem to the continuous setting we begin with a parameter $\beta \in(0,1 / 2)$ which will play the role of $q$ and define a collection $M_{\beta}$ of images $F \in L^{2}\left([0,1]^{2}\right)$ as follows: we write $F \in M_{\beta}$ if $F$ is supported by a compact set $K \subset[0,1]^{2}$ whose measure $|K|$ does not exceed $\beta$. This compact set $K$ depends on $F$ and $M_{\beta}$ is not a vector space. If $F, G$ belong to $M_{\beta}$, then $F+G$ belongs to $M_{2 \beta}$, a situation which is classical in nonlinear approximation. It will be proved below that for every $\alpha \in(0,1 / 2)$ there exists a set $\Lambda_{\alpha} \subset \mathbb{Z}^{2}$ with the following properties: (a) density $\Lambda_{\alpha}=2 \alpha$ and (b) the mapping $\Phi: M_{\beta} \mapsto \ell^{2}\left(\Lambda_{\alpha}\right)$ defined by $\Phi(F)=(\hat{F}(k))_{k \in \Lambda_{\alpha}}$ is injective when $0<\beta<\alpha$. This set $\Lambda_{\alpha}$ plays the role of $\Omega$ in Tao's work and the density of $\Lambda_{\alpha}$ is then playing the role of the cardinality of $\Omega$. Any $F \in M_{\beta}$ can be retrieved from the information given by the "irregular sampling" $\hat{F}(k)=a(k), k \in \Lambda_{\alpha}$, and one would like to do it by some fast algorithm. If we a priori know that the data $a(k), k \in \Lambda_{\alpha}$, are the Fourier coefficients of some nonnegative $F \in M_{\beta}$, then it will be proved that $F$ is the unique solution of the following problem

$$
\inf \left\{\|u\|_{1} ; u \in L^{1}\left(\mathbb{T}^{2}\right), \hat{u}(k)=a(k), k \in \Lambda_{\alpha}\right\} .
$$

The reader recognizes the minimization algorithm which is used in compressed sensing. We do not impose any condition on the support of $u$ in (1.2). The uniqueness of the solution of the problem (1.2) is coming from the peculiar structure of the data $a(k), k \in \Lambda_{\alpha}$. This is no longer true when $F$ both takes positive and negative values (see Lemma 6.2). We now construct the sparse set $\Lambda_{\alpha}$.

Definition 1.1. If $\alpha \in(0,1 / 2)$ we define $\Lambda_{\alpha} \subset \mathbb{Z}^{2}$ by

$$
\Lambda_{\alpha}=\left\{(m, n) \in \mathbb{Z}^{2} ; \exists r \in \mathbb{Z} \text { such that }|m \sqrt{2}+n \sqrt{3}-r| \leq \alpha\right\} .
$$

The choice of $\sqrt{2}$ and $\sqrt{3}$ is irrelevant and other irrational numbers $\gamma_{1}$ and $\gamma_{2}$ could be used as long as $\gamma_{1}, \gamma_{2}$ and 1 are linearly independent over $\mathbb{Q}$. We know from the theory of "model sets" [4] that the density of $\Lambda_{\alpha} \subset \mathbb{Z}^{2}$ is uniform and equals $2 \alpha$. It means that for every $\varepsilon>0$ there exists a $R(\varepsilon)$ such that for $R \geq R(\varepsilon)$ and uniformly in $x_{0} \in \mathbb{Z}^{2}$

$$
(2 \alpha-\varepsilon) \pi R^{2} \leq \#\left\{\Lambda_{\alpha} \cap B\left(x_{0}, R\right)\right\} \leq(2 \alpha+\varepsilon) \pi R^{2} .
$$

Here $B\left(x_{0}, R\right)$ is the disc centered at $x_{0}$ with radius $R$.

Definition 1.2. As above we write $F \in M_{\beta}$ if $F \in L^{2}\left(\mathbb{T}^{2}\right)$ and if $F$ is supported by a compact set $K \subset[0,1]^{2}$ whose measure $|K|$ does not exceed $\beta$. 
Theorem 1.1. We assume $\beta<\alpha$ and we have

(a) The mapping $\Phi: M_{\beta} \mapsto l^{2}\left(\Lambda_{\alpha}\right)$ defined by $F \mapsto(\hat{F}(k))_{k \in \Lambda_{\alpha}}$ is injective.

(b) If $F \in M_{\beta}$ is nonnegative, then $F$ is the unique solution $u$ of the following variational problem

$$
\inf \left\{\|u\|_{1} ; u \in L^{1}\left(\mathbb{T}^{2}\right), \hat{u}(k)=\hat{F}(k), k \in \Lambda_{\alpha}\right\} .
$$

(c) If $F \in M_{\beta}, u \in L^{1}\left(\mathbb{T}^{2}\right), F \geq 0, u \geq 0$ and $\hat{u}(k)=\hat{F}(k), k \in \Lambda_{\alpha}$, then $u=F$.

Let us stress that we do not assume anything on the support of $u$ in (1.5). Theorem 1.1 is sharp since the hypothesis $\beta<\alpha$ cannot be replaced by $\beta>\alpha$. The case $\beta=\alpha$ seems to be open. If one does not assume $F \geq 0$ then $F$ is not in general the argument of the variational problem (1.5). These two remarks will be proved later on (see Section 6). We also want to investigate the stability in Theorem 1.1 which is given by the following statement where the error term is measured in $L^{\infty}\left(\mathbb{T}^{2}\right)$. It would have been more natural to measure error terms in $L^{2}$ but we were unable to prove Theorem 1.2 in this context.

Theorem 1.2. Let us assume we are given a noisy sampling $a(k)=\hat{F}(k)+$ $\hat{R}(k), k \in \Lambda_{\alpha}$, where the unknown function $F$ is nonnegative and belongs to $M_{\beta}$ and where the error term $R$ fulfils $\|R\|_{\infty} \leq \varepsilon$. Then for any nonnegative $u$, the property $\hat{u}(k)=a(k), k \in \Lambda_{\alpha}$, implies $\|u-F\|_{\infty} \leq C \varepsilon$ where $C=C(F)$ only depends on the geometry of the closed support of $F$.

Let us say a few words on $C(F)$. One might hope that $C(F)$ be a function of $\alpha$ and $\beta$. We have not been able to prove it. Indeed in our proof $C(F)$ will depend on a function $\beta(\varepsilon)$ which is the measure of the sum $K+B(0, \varepsilon)$ between $K$ ( $K$ is the closed support of $F$ ) and the disc centered at 0 with radius $\varepsilon$. The behavior of $\beta(\varepsilon)$ near 0 depends on the geometry of $K$. This will be detailed in Section 4 (see Proposition 4.2).

We now say a few words on the organization of this paper. Following H. J. Landau, A. Olevskii and A. Ulanovskii, we study two properties named "stable sampling" and "stable interpolation" in Sections 2 and 3. Up to some obvious transformation, sampling and interpolation are proved to be equivalent properties (see Proposition 2.1). This permits to focus on stable interpolation in the proof of Theorem 1.1. Stable interpolation will follow from Proposition 4.1 which is seminal in this paper. Then the proofs of Theorems 1.1 and 1.2 will be completed in Section 4 and 5 and the last section will be devoted to some counterexamples. We decided to focus on 
the two-dimensional case but the theorems and proofs obviously extend to any dimension. We conclude this introduction with a few words on image processing. A cartoon image is defined by a function $F$ which is piecewise constant with jump discontinuities along a finite collection $K$ of rectifiable curves. Then $\nabla F$ is supported by $K$ and the measure of $K$ is 0 . Then for any positive $\alpha$, the subsampling $\hat{F}(k), k \in \Lambda_{\alpha}$, suffices to recover $F$. Indeed the Fourier transform of $\nabla F$ is given by $i k \hat{F}(k)$ and Theorem 1.1 implies our claim. One could object that Theorem 1.1 is restricted to the $L^{2}$ case while $\nabla F$ is a Radon measure. This issue is not a serious one and the details of the argument will appear elsewhere.

\section{2. $L^{2}$ and $L^{p}$ estimates}

We return to the finite field $\mathbb{F}_{p}$ and assume that we are given two sets $T \subset \mathbb{F}_{p}$ and $\Omega \subset \mathbb{F}_{p}$ with the same cardinality. We denote by $l_{T}^{2}$ the linear space of all $f \in l^{2}$ which are supported by $T$ (i.e. vanish outside $T$ ). Similarly $l^{2}(\Omega)$ denotes the restrictions to $\Omega$ of signals $f \in l^{2}$. This looks pedantic but will be adapted to functional spaces in a continuous setting. The theorem by Tao can be split into two pieces. The first assertion says that the mapping $\mathcal{F}: l_{T}^{2} \mapsto l^{2}(\Omega)$ defined by $f \mapsto \hat{f}$ is an isomorphism. The second assertion in Tao's theorem says that the mapping $\mathcal{F}: M_{q} \mapsto l^{2}(\Omega)$ is injective when $\# \Omega \geq 2 q$. Here $T$ is not given. This obviously follows from the first statement. It suffices to observe that $f_{1}, f_{2} \in M_{q}$ implies $f=f_{1}-f_{2} \in M_{2 q}$. We aim at generalizing these two facts to the continuous setting. Let us begin with the case where the set $T$ is given. Let $K \subset \mathbb{T}^{2}$ be a Borel set ( $K$ will play the role of $T$ ) and $\Lambda \subset \mathbb{Z}^{2}$ be a set of frequencies ( $\Lambda$ will play the role of $\Omega$ ). Let $L_{K}^{2}$ be the Hilbert space of all square integrable functions supported by $K$. In other words we write $F \in L_{K}^{2}$ if $F$ belongs to $L^{2}\left(\mathbb{T}^{2}\right)$ and vanishes almost everywhere on $\mathbb{T}^{2} \backslash K$. H. J. Landau defined in [3] a set of stable sampling by the following condition

Definition 2.1. Let $K \subset \mathbb{T}^{2}$ be a Borel set and $\Lambda \subset \mathbb{Z}^{2}$ a set of frequencies. We say that $\Lambda$ is a set of stable sampling for $L_{K}^{2}$ if a constant $C$ exists such that for every $F \in L_{K}^{2}$ one has

$$
\|F\|_{2} \leq C\left(\sum_{k \in \Lambda}|\hat{F}(k)|^{2}\right)^{1 / 2}
$$

Here and in what follows, $\hat{F}(k), k \in \mathbb{Z}^{2}$, denotes the sequence of Fourier coefficients of $F \in L^{1}\left(\mathbb{T}^{2}\right)$. In other words $\Lambda$ is a set of stable sampling for $L_{K}^{2}$ if and only if the functions $\exp (2 \pi i k \cdot x), k \in \Lambda$, are a frame of $L^{2}(K)$. Then 
the measure $|K|$ of $K$ cannot exceed the lower density of $\Lambda$ as H. J. Landau proved in [3].

Definition 2.2. We say that $\Lambda \subset \mathbb{Z}^{2}$ is a universal sampling set if the following conditions hold: $\Lambda$ has a uniform density d and, for every compact set $K$ of Lebesgue measure less that $d, \Lambda$ is a set of stable sampling for $L_{K}^{2}$.

As it was already mentioned, H. J. Landau proved that the condition $|K| \leq d$ is necessary. In the definition of universal sampling sets $K$ is assumed to be a compact set. A. Olevskii and A. Ulanovskii who defined universal sampling sets proved in [7] that universal sampling sets would not exist if $K$ was allowed to be an arbitrary Borel set of measure less than $d$. We aim at constructing universal sampling sets. The most convenient way relies on the property of stable interpolation which was also introduced by H. J. Landau:

Definition 2.3. Let $\Omega \subset \mathbb{T}^{2}$ a Borel set. We say that $\Lambda \subset \mathbb{T}^{2}$ is a set of stable interpolation for $L^{2}(\Omega)$ if for every $(a(\lambda))_{\lambda \in \Lambda} \in l^{2}(\Lambda)$ there exists a $F \in L_{\Omega}^{2}$ such that $\hat{F}(\lambda)=a(\lambda), \lambda \in \Lambda$.

This definition can be given an equivalent form :

Lemma 2.1. $\Lambda \subset \mathbb{Z}^{2}$ is a set of stable interpolation for $L^{2}(\Omega)$ if and only if there exists a constant $C$ such that for any square summable sequence $(a(k))_{k \in \Lambda}$ one has

$$
\left(\sum_{k \in \Lambda}|a(k)|^{2}\right)^{1 / 2} \leq C\left(\int_{\Omega}\left|\sum_{k \in \Lambda} a(k) \exp (2 \pi i k \cdot x)\right|^{2} d x\right)^{1 / 2}
$$

If $\Lambda \subset \mathbb{Z}^{2}$ is a set of stable sampling and at the same time a set of stable interpolation for $L^{2}(\Omega)$ then the functions $\exp (2 \pi i k \cdot x), k \in \Lambda$, are a Riesz basis for $L^{2}(\Omega)$. Finding necessary and sufficient conditions for (2.1) or (2.2) is out of reach and only necessary conditions are known. These necessary conditions were obtained by H. J. Landau in [3]. In these conditions the upper or lower density of $\Lambda$ are compared to the measure of $\Omega$ or $K$.

Definition 2.4. The upper density $D^{+}(\Lambda)$ of $\Lambda$ is defined as

$$
\limsup _{R \rightarrow \infty} \sup _{x} \frac{\#\{B(x, R) \cap \Lambda\}}{\pi R^{2}}
$$

and the lower density $D_{-}(\Lambda)$ is defined by

$$
\liminf _{R \rightarrow \infty} \inf _{x} \frac{\#\{B(x, R) \cap \Lambda\}}{\pi R^{2}} .
$$


H. J. Landau proved the implications $(2.2) \Rightarrow D^{+}(\Lambda) \leq|\Omega|$ and $(2.1) \Rightarrow$ $D_{-}(\Lambda) \geq|K|$. These necessary conditions are obviously not sufficient.

Sampling and interpolation are the same problem as it will be proved now.

Proposition 2.1. If the Borel set $K$ is the complement of $\Omega$ in $\mathbb{T}^{2}$ and if $M$ is the complement of $\Lambda$ in $\mathbb{Z}^{2}$, then $M$ is a set of stable sampling for $L_{K}^{2}$ if and only if $\Lambda$ is a set of stable interpolation for $L^{2}(\Omega)$.

For proving $(2.2) \Rightarrow(2.1)$ we start with a function $F \in L^{2}\left(\mathbb{T}^{2}\right)$ which is supported by $K$ and we need to prove that $\|F\|_{2} \leq C\left(\sum_{k \in M}|\hat{F}(k)|^{2}\right)^{1 / 2}$. We then split $f=\hat{F}$ into the sum $g+h$ where $g=f \mathbf{1}_{M}$ and $h=f \mathbf{1}_{\Lambda}$. Here and in what follows $\mathbf{1}_{E}$ denotes the indicator function of the set $E$. Then $g(k), k \in \mathbb{Z}^{2}$, are the Fourier coefficients of $G \in L^{2}\left(\mathbb{T}^{2}\right)$ and $h(k), k \in \mathbb{Z}^{2}$, are the Fourier coefficients of $H \in L^{2}\left(\mathbb{T}^{2}\right)$. We have $F=G+H$. From (2.2) we know that

$$
\|h\|_{2} \leq C\left(\int_{\Omega}|H(x)|^{2} d x\right)^{1 / 2}
$$

But $G+H=0$ on $\Omega$ and

$$
\int_{\Omega}|H(x)|^{2} d x=\int_{\Omega}|G(x)|^{2} d x \leq\|G\|_{2}^{2}=\|g\|_{2}^{2}=\sum_{k \in M}|f(k)|^{2} .
$$

Therefore

$$
\|h\|_{2} \leq C\left(\sum_{k \in M}|f(k)|^{2}\right)^{1 / 2}
$$

and

$$
\|f\|_{2}=\left(\|g\|_{2}^{2}+\|h\|_{2}^{2}\right)^{1 / 2} \leq \sqrt{1+C^{2}}\left(\sum_{k \in M}|f(k)|^{2}\right)^{1 / 2}
$$

which ends the proof.

The converse implication $(2.1) \Rightarrow(2.2)$ is just as easy. We assume that $f(k)$ vanishes outside $\Lambda$ and we consider $F(x)=\sum_{k \in \Lambda} f(k) \exp (2 \pi i k \cdot x)$. We want to prove (2.2). We split $F(x)$ into the sum $G(x)+H(x)$ where $G$ is the product between $F$ and the indicator function $\mathbf{1}_{K}$ of $K$ and $H(x)=$ $\mathbf{1}_{\Omega} F(x)$. Let $g(k)$ and $h(k), k \in \mathbb{Z}^{2}$, be the Fourier coefficients of $G$ and $H$. We know that $f=g+h$ and we have $f(k)=0$ whenever $k \in M$. Then (2.1) yields $\|G\|_{2}^{2} \leq C^{2} \sum_{k \in M}|g(k)|^{2}$. But $g(k)=-h(k), k \in M$, and $\|G\|_{2}^{2} \leq C^{2} \sum_{k \in M}|h(k)|^{2} \leq C^{2}\|H\|_{2}^{2}=C^{2} \int_{\Omega}|F|^{2} d x$ which ends the proof. Indeed $\|F\|_{2}^{2}=\|G\|_{2}^{2}+\|H\|_{2}^{2}$ implies $\|F\|_{2} \leq \sqrt{1+C^{2}}\|H\|_{2}$. 
Definition 2.5. We say that $\Lambda$ is a set of universal interpolation if $\Lambda$ has a uniform density $d$ and is a set of stable interpolation for any open set $\Omega$ of measure larger than $d$.

A. Olevskii and A. Ulanovskii proved in [7] that universal interpolation sets would not exist if $\Omega$ was allowed to be an arbitrary Borel set of measure larger than $d$.

We aim at proving the following theorem

Theorem 2.1. The set $\Lambda_{\alpha}$ of Definition 1.1 is a set of universal sampling and also of universal interpolation.

Theorem 2.1 can be traced back to [4] where it is proved for functions of one real variable (see Theorem 6, page 51). This little book was published in 1970 in French and remained unnoticed. A. Olevskii and A. Ulanovskii were not aware of [4] and independently constructed sets of stable sampling and sets of stable interpolation. For functions of one real variable, they proved the following

Theorem 2.2. For every positive $d$ and $\varepsilon$ there exists a sequence $\Lambda \subset \mathbb{R}$ satisfying the following conditions

(a) $\|\Lambda-(1 / d) \mathbb{Z}\|_{\infty}<\varepsilon$

(b) the family $\exp (i \lambda x), \lambda \in \Lambda$, is a frame in $L^{2}(S)$ for every compact set $S$ of measure $<2 \pi d$.

(c) for every open set $S$ of measure $>2 \pi d$, any square summable sequence $a(\lambda), \lambda \in \Lambda$, is the restriction to $\Lambda$ of the Fourier transform of a function $F \in L^{2}(S)$.

In this theorem, the Fourier transform is defined without $2 \pi$ in the exponential. Theorem 2.1 implies (a) in Theorem 1.1. Indeed if $F_{1}, F_{2} \in M_{\beta}$, then $F=F_{1}-F_{2}$ is supported by a compact set $K$ of measure less than $2 \beta$. If $\hat{F}_{1}(k)=\hat{F}_{2}(k), k \in \Lambda_{\alpha}$, we have $\hat{F}(k)=0, k \in \Lambda_{\alpha}$ and Theorem 2.1 implies $F=0$. The proof of Theorem 2.1 will be given in Section 4. This proof relies on a generalization of sets of stable interpolation where $L^{2}$ estimates are replaced by $L^{\infty}$ estimates. These $L^{\infty}$ estimates will be obtained by transference. The proof of the universal sampling property will then follow from Proposition 2.1, Proposition 2.2 and from the fact that the complement of $\Lambda_{\alpha}$ has a similar structure. The details of this proof are given in Section 4. The $L^{\infty}$ estimates are defined now. It does not cost more to treat the general case of an exponent $p \in[1, \infty]$. Let $K \subset \mathbb{T}^{2}$ be a Borel set. We define $L_{K}^{p}$ as the Banach space of all $F \in L^{p}$ which are supported 
by $K$. The space $L^{p}(\Omega)$ will consist of all restrictions to $\Omega$ of functions in $L^{p}$. This looks pedantic since $L^{p}(K)$ and $L_{K}^{p}$ are identical. But a function in $L_{K}^{p}$ needs to be viewed as a function which vanishes outside $K$. Let $E^{p}$ be the Banach space of Fourier coefficients $\hat{F}(k), k \in \mathbb{Z}^{2}$ of functions $F$ in $L^{p}\left(\mathbb{T}^{2}\right)$, a Banach space that some authors denote by $\mathcal{F} L^{p}$. For $\Lambda \subset \mathbb{Z}^{2}$, we define the Banach space $E^{p}(\Lambda)$ as the space of restrictions to $\Lambda$ of all $f \in E^{p}$ and we equip $E^{p}(\Lambda)$ with the quotient norm. We now define a set of stable sampling for $E_{K}^{p}$.

Definition 2.6. We say that $\Lambda$ is a set of stable sampling for $L_{K}^{p}$ if there exists a constant $C$ such that one has $\|F\|_{p} \leq C\|\hat{F}\|_{E^{p}(\Lambda)}$ for every $F \in L_{K}^{p}$.

In other words $F \in L^{p}\left(\mathbb{T}^{2}\right), F(x)=0$ almost everywhere on $\mathbb{T}^{2} \backslash K$, $G \in L^{p}\left(\mathbb{T}^{2}\right)$, and $\hat{F}(k)=\hat{G}(k), k \in \Lambda$, imply $\|F\|_{p} \leq C\|G\|_{p}$. This is the most convenient formulation. If $1 \leq p<\infty$ and $1 / p+1 / q=1$, then Definition 2.6 is equivalent to the following condition

Lemma 2.2. A set of frequencies $\Lambda$ is a set of stable sampling for $L_{K}^{p}$ if and only if every $F \in L^{q}(K)$ is the restriction to $K$ of a function $G(x)=$ $\sum_{k \in \Lambda} a(k) \exp (2 \pi i k \cdot x)$ which belongs to $L^{q}\left(\mathbb{T}^{2}\right)$.

We now define a set of stable interpolation for $L_{K}^{p}$. Let us denote by $\mathcal{C}_{\Lambda}$ the space of all continuous functions $F$ on $\mathbb{T}^{2}$ such that $\hat{F}(k)=0$ if $k \notin \Lambda$. We notice that $(2.1)$ can be written $F \in \mathcal{C}_{\Lambda} \Rightarrow\|F\|_{2} \leq C\|F\|_{L^{2}(\Omega)}$. This observation leads to the following definition

Definition 2.7. Let $\Omega \subset \mathbb{T}^{2}$ be a Borel set and $p \in[1, \infty]$. We say that $\Lambda$ is a set of stable interpolation for $L^{p}(\Omega)$ if

$$
F \in \mathcal{C}_{\Lambda} \Rightarrow\|F\|_{p} \leq C\|F\|_{L^{p}(\Omega)}
$$

When $p=2$ this new definition is the one we gave above. If $1 / p+1 / q=1$ a duality argument yields the following lemma

Lemma 2.3. $\Lambda$ is a set of stable interpolation for $L_{K}^{p}$ if and only if each sequence $(a(k))_{k \in \Lambda}$ belonging to $E^{q}(\Lambda)$ is the restriction to $\Lambda$ of the Fourier coefficients of $F \in L_{K}^{q}$.

If $p=1$ or $p=\infty$ this statement needs to be modified accordingly. Keeping these definitions in mind we have

Theorem 2.3. As in Proposition 2.1, let $M \subset \mathbb{Z}^{2}$, let $\Lambda$ be the complement of $M$ in $\mathbb{Z}^{2}$ and let $K \subset \mathbb{T}^{2}$ be a Borel set. Let $\Omega$ be the compement of $K$ in $\mathbb{T}^{2}$. If $M$ is a set of stable sampling for $L_{K}^{p}$, then $\Lambda$ is a set of stable interpolation for $L_{\Omega}^{p}$. Conversely if $\Lambda$ is a set of stable interpolation for $L_{\Omega}^{p}$ and if the indicator function of $\Lambda$ is a multiplier for $\mathcal{F} L^{p}$, then $M$ is a set of stable sampling for $L_{K}^{p}$. 
Before proving this theorem, let us observe that the set $\Lambda_{\alpha}$ which is defined by (1.3) has the required property. This follows from the fact that the indicator function of an interval is a multiplier for $\mathcal{F} L^{p}$ and from the transference methods of R. Coifman and G. Weiss. The proof of Theorem 2.3 is similar to the one we gave for Proposition 2.1. We assume that $M$ is a set of stable sampling for $L_{K}^{p}$ and we want to prove (2.7) when $F \in \mathcal{C}_{\Lambda}$. We have $F(x)=\sum_{k \in \Lambda} f(k) \exp (2 \pi i k \cdot x)$. We split $F(x)$ into the sum $G(x)+H(x)$ where $G$ is the product between $F$ and the indicator function of $K$ and $H(x)=\mathbf{1}_{\Omega} F(x)$. Let $g(k)$ and $h(k), k \in \mathbb{Z}^{2}$, be the Fourier coefficients of $G$ and $H$. We know that $f=g+h$ and we have $f(k)=0$ whenever $k \in M$. Then $g=-h$ on $M$ and the equivalent definition of a set of stable sampling yields $\|G\|_{p} \leq C\|H\|_{p}=C\|F\|_{L^{p}(\Omega)}$. The proof of (2.7) ends with $\|F\|_{p} \leq\|G\|_{p}+\|H\|_{p} \leq(1+C)\|F\|_{L^{p}(\Omega)}$.

The converse implication is as easy. We start with two functions $F$ and $G$ such that $F$ is supported by $K$ and $\hat{F}=\hat{G}$ on $M$. We want to prove that $\|F\|_{p} \leq C\|G\|_{p}$. For proving it we denote by $f(k)$ the Fourier coefficients of $F$ and by $g(k)$ the Fourier coefficients of $G$. We write $F=F_{1}+F_{2}$ where $F_{1}(x)=\sum_{k \in M} f(k) \exp (2 \pi i k \cdot x)=\sum_{k \in M} g(k) \exp (2 \pi i k \cdot x)$ and $F_{2}(x)=\sum_{k \in \Lambda} f(k) \exp (2 \pi i k \cdot x)$. Since $\Lambda$ is a set of stable interpolation for $E_{\Omega}^{p}$ we obtain $\left\|F_{2}\right\|_{p} \leq C\left\|F_{2}\right\|_{L^{p}(\Omega)}$. But $F_{1}+F_{2}=0$ on $\Omega$ which implies $\left\|F_{2}\right\|_{L^{p}(\Omega)}=\left\|F_{1}\right\|_{L^{p}(\Omega)} \leq\left\|F_{1}\right\|_{p}$. Finally the Fourier coefficients of $F_{1}$ are given by $f_{1}(k)=f(k)=g(k), k \in M$, and 0 elsewhere. If $\mathbf{1}_{M}$ is a multiplier of $\mathcal{F} L^{p}$, we have $\left\|F_{1}\right\|_{p} \leq C\|G\|_{p}$ which ends the proof.

The following result will be in seminal in the construction of sets of stable interpolation.

Proposition 2.2. Let $\Omega \subset \mathbb{T}^{2}$ be an open set containing a compact set $K$. Let us assume that $q \geq p$ and that $\Lambda \subset \mathbb{Z}^{2}$ is a set of stable interpolation for $E_{K}^{q}$. Then $\Lambda$ is a set of stable interpolation for $E_{\Omega}^{p}$.

The proof is not difficult and will be detailed if $q=\infty$ and $p=2$. Let one denote by $B(0, \varepsilon)$ the ball centered at 0 with radius $\varepsilon$ where $\varepsilon$ is fixed such that $K+B(0, \varepsilon) \subset \Omega$. Next one denotes by $g \in L^{2}(B(0, \varepsilon))$ an arbitrary function satisfying $\|g\|_{2} \leq 1$. Then if $\|F\|_{L^{2}(\Omega)} \leq 1$, one has $\|F * g\|_{L^{\infty}(K)} \leq 1$ and the spectrum of $F * g$ is included in that of $F$. Then (2.7) implies $\|F * g\|_{\infty} \leq C$, i.e. $\left|\int F(x) g\left(x_{0}-x\right) d x\right| \leq C$, for every $x_{0}$. Optimizing in $g$ one obtains

$$
\left(\int_{B\left(x_{0}, \varepsilon\right)}|F(x)|^{2} d x\right)^{1 / 2} \leq C .
$$

It suffices to cover $\mathbb{T}^{2}$ with $\varepsilon^{-2}$ such discs to obtain $\|F\|_{2} \leq C \varepsilon^{-1}$. The same proof shows that a set of stable interpolation for $E_{K}^{q}$ is a set of stable interpolation for $E_{\Omega}^{p}$ when $p \leq q$. 
The following theorem will be proved in Section 4 .

Theorem 2.4. If $\leq p \leq \infty$ and if the measure of the compact set $K$ is less than $2 \alpha$, then the set $\Lambda_{\alpha}$ of Theorem 2.1 is a set of stable sampling for $L^{p}(K)$.

Let us stress that $2 \alpha$ is the density of $\Lambda_{\alpha}$.

\section{Extension to $l^{p}-$ norms}

The extension to $l^{p}$-norms, $p \in[1, \infty]$, of the definition of a set of stable sampling will be discussed in this section. The reader may wish to skip this section which is not needed in the proof of Theorem 2.1. Let us assume that $K \subset \mathbb{T}^{2}$ is a compact set. Given an exponent $p$ we would like to know whether or not there exists a constant $C=C(K, M, p)$ such that

$$
\left(\sum_{k \in \mathbb{Z}^{2}}|f(k)|^{p}\right)^{1 / p} \leq C\left(\sum_{k \in M}|f(k)|^{p}\right)^{1 / p}
$$

whenever $f(k)$ are the of Fourier coefficients of a function or a distribution $F$ supported by $K$. Let us stress that in general $F$ is no longer a function when $p>2$. Therefore the support of $F$ is the closed support of a distribution. That is why $K$ is closed in (3.1). The estimate (3.1) says that $M$ is a set of stable sampling for a space that we define now. We let $Y^{p}$ be the Banach space consisting of the functions or distributions (when $p>2$ ) whose Fourier coefficients belong to $l^{p}$. Then the left-hand side of (3.1) is the norm in $Y^{p}$ of $F(x)=\sum_{k \in \mathbb{Z}^{2}} f(k) \exp (2 \pi i k \cdot x)$.

Definition 3.1. Let $Y_{K}^{p}$ denote the space of all functions or distributions in $Y^{p}$ which are supported by $K$. If (3.1) holds for every $F \in Y_{K}^{p}$ we say that $M$ is a set of stable sampling for the space $Y_{K}^{p}$.

Let now $\Omega \subset \mathbb{T}^{2}$ be an open set. We define the Banach space $Y^{p}(\Omega)$ as the space of restrictions to $\Omega$ of all generalized functions $F \in Y^{p}$, the norm being the obvious quotient norm.

Definition 3.2. A set $\Lambda$ of stable interpolation for $Y^{p}(\Omega)$ is defined by the existence of a constant $C$ such that $F \in \mathcal{C}_{\Lambda} \Rightarrow\|F\|_{Y^{p}} \leq C\|F\|_{Y^{p}(\Omega)}$.

We then have

Proposition 3.1. Let $1 \leq p \leq \infty, \Omega \subset \mathbb{T}^{2}$ be an open set and $K$ a compact set contained in $\Omega$. If we have $F \in \mathcal{C}_{\Lambda} \Rightarrow\|F\|_{\infty} \leq C\|F\|_{L^{\infty}(K)}$, then $F \in$ $\mathcal{C}_{\Lambda} \Rightarrow\|F\|_{p} \leq C\|F\|_{Y^{p}(\Omega)}$. Moreover the complement $M$ of $\Lambda$ in $\mathbb{T}^{2}$ is a set of stable sampling for $Y_{R}^{p}$ where $R$ is the complement of $\Omega$ in $\mathbb{T}^{2}$. 
The proof of the second statement ("interpolation implies sampling for the complementary set") is similar to the one we gave in the $L^{2}$ setting (see Proposition 2.1) and we leave the details to the reader. We now prove the first statement in Proposition 3.1. First we observe that $Y^{p}$ is the dual of $Y^{p^{\prime}}$ when $p$ and $p^{\prime}$ are conjugate exponents (with an obvious modification if $p=1$ ). Moreover a function on $\mathbb{T}^{2}$ belongs to $Y^{p}$ if and only if it locally belongs to $Y^{p}$. We assume that $\varepsilon$ is small enough to ensure $K+B(0, \varepsilon) \subset \Omega$. We then pick a test function $g$ supported in $B(0, \varepsilon)$ and belonging to the unit ball of $Y^{p^{\prime}}$. If $\|F\|_{Y^{p}(\Omega)} \leq 1$, then $\|F * g\|_{L^{\infty}(K)} \leq 1$. We then use the hypothesis to obtain $\|F * g\|_{\infty} \leq C$. We optimize in $g$ as we did before. Then all local $Y^{p}$ norms of $F$ are controlled. This implies the required estimate on the full $Y^{p}$ norm of $F$.

\section{A proof of Theorem 2.1}

We now prove Theorem 2.1. An alternative proof of Theorem 2.1 can be found in [4] (pages 39 to 50). As was already announced, the proof of the $L^{\infty}$ interpolation property (Proposition 4.1) is the easiest one and will be obtained by transference. We then use Proposition 2.2 to obtain the interpolation property and finally the sampling property will result from Proposition 2.1.

We now introduce the tools which will be used for the transference method. Let us define $\Pi: \mathbb{R} \mapsto \mathbb{T}=\mathbb{R} / \mathbb{Z}$ by $\Pi(t)=t(\bmod 1)$. Then $\mathbb{Z}^{2}$ can be embedded in $\mathbb{T}$ by $\gamma^{*}: \mathbb{Z}^{2} \mapsto \mathbb{T}$ which is defined by

$$
\gamma^{*}(m, n)=\Pi(m \sqrt{2}+n \sqrt{3})
$$

This mapping $\gamma^{*}$ is injective with a dense range. With an obvious abuse of notations we still denote by $\Pi$ the canonical mapping from $\mathbb{R}^{2}$ to $\mathbb{T}^{2}$. Then the dual mapping $\gamma: \mathbb{Z} \mapsto \mathbb{T}^{2}$ is given by $\gamma(k)=\Pi(k \sqrt{2}, k \sqrt{3})$ and the range of $\gamma$ will be denoted by $\Gamma$. Then $\Gamma$ is dense in $\mathbb{T}^{2}$.

One denotes by $I \subset \mathbb{T}$ an arbitrary interval (or arc) of the circle. This arc is not necessarily centered in 0 and the complement of $I$ in $\mathbb{T}$ is also an interval. Then we define $\Lambda_{I} \subset \mathbb{Z}^{2}$ by

$$
\Lambda_{I}=\left\{(m, n) \in \mathbb{Z}^{2} ; \gamma^{*}(m, n) \in I\right\} .
$$

If $I=[a, b]$ where $0<a<b<1, \Lambda_{I}=\left\{(m, n) \in \mathbb{Z}^{2} ; \exists r \in \mathbb{Z}\right.$ such that $a \leq$ $m \sqrt{2}+n \sqrt{3}-r \leq b\}$. The density of $\Lambda_{I}$ is uniform and equals $|I|$. A compact set $K \subset \mathbb{T}^{2}$ is Riemann integrable if the measure of the boundary of $K$ is 0 . Let us then define $M_{K}$ as the set of all $k \in \mathbb{Z}$ such as $\gamma(k) \in K$. Then the density of $M_{K}$ is uniform and equals the Lebesgue measure $|K|$ of $K$ as it 
is proved in [4]. As it was announced the following result is seminal in the proof of Theorem 2.1 .

Proposition 4.1. Let us assume that $\Omega$ is an open set of measure $|\Omega|>|I|$. Then $\Lambda_{I}$ is a set of stable interpolation for $E_{\Omega}^{\infty}$.

In other words there exists a constant $C=C_{\Omega, I}$ such that for any function $F \in \mathcal{C}\left(\mathbb{T}^{2}\right)$ whose Fourier coefficients vanish outside $\Lambda_{I}$ one has

$$
\|F\|_{\infty} \leq C \sup _{x \in \Omega}|F(x)|
$$

Before proving it, let us observe that one cannot replace the open set $\Omega$ by a compact set $K$ in Proposition 4.1. Indeed $|K|>|I|$ does not suffice to obtain (4.3).

We now prove Proposition 4.1. For proving (4.3) it suffices to assume that $F$ is a finite trigonometrical sum. Then one has

$$
F\left(x_{1}, x_{2}\right)=\sum_{(m, n) \in \Lambda_{I}} a_{(m, n)} e^{2 \pi i\left(m x_{1}+n x_{2}\right)} .
$$

It implies

$$
F(k \sqrt{2}, k \sqrt{3})=\sum_{(m, n) \in \Lambda_{I}} a_{(m, n)} e^{2 \pi i k(m \sqrt{2}+n \sqrt{3})}=\hat{\mu}(-k)
$$

where $\mu$ is the measure on $\mathbb{T}$ which is the sum of the Dirac masses $a_{(m, n)}$ at the points $m \sqrt{2}+n \sqrt{3}$ which belong to $I$, modulo 1 . If $\Pi(k \sqrt{2}, k \sqrt{3}) \in \Omega$ then $k \in M_{\Omega}$. Since $\Gamma=\gamma(\mathbb{Z})$ is dense in $\mathbb{T}^{2}$ we have

$$
\sup _{\Omega}|F|=\sup _{k \in M_{\Omega}}|\hat{\mu}(-k)| \text {. }
$$

Before stating our next lemma we return to the definition of the lower density. Here and in Lemma $4.1, M \subset \mathbb{Z}$ is any set of integers.

Definition 4.1. The lower density $D_{-}(M)$ of $M \subset \mathbb{Z}$ is the upper bound of the set of nonnegative numbers $d$ such that for every $\varepsilon>0$ there exists a $R(\varepsilon)$ such that for $R \geq R(\varepsilon)$ we have, uniformly in $m \in \mathbb{Z}$,

$$
d(R-\varepsilon) \leq \#\{M \cap[m, m+R]\}
$$

Proposition 4.1 is now an easy consequence of the following lemma 
Lemma 4.1. Let $M \subset \mathbb{Z}$ be any set of integers and let $d$ be the lower density of $M$. If $|I|$ denotes the length of the arc $I \subset \mathbb{T}$ and if $d>|I|$, then there exists a constant $C=C(M, I)$ such that

$$
\sup _{k \in \mathbb{Z}}|\hat{\mu}(k)| \leq C \sup _{k \in M}|\hat{\mu}(k)|
$$

for any measure $\mu$ carried by I. If we are given a positive number $d$ and a sequence $M_{j}$ of sets of integers for which (4.7) holds uniformly in $j$, and if $d>|I|$, then we have $C\left(M_{j}, I\right) \leq C$.

A proof of Lemma 4.1 can be found in [4]. Let us sketch the argument for the reader's convenience. The fact that $\mu$ is a measure does not play any role. Indeed, Lemma 4.1 remains true if $\mu$ is replaced by a pseudomeasure $\sigma$ carried by $I$. A pseudo-measure is a distribution whose Fourier transform belongs to $l^{\infty}(\mathbb{Z})$. The proof of Lemma 4.1 relies on the following observations. If $M_{j} \subset \mathbb{Z}, j \in \mathbb{N}$, is a sequence of sets of integers, we say that $M_{j}$ weakly converges to $M$ if for each integer $R$, we have $M \cap[-R, R]=$ $M_{j} \cap[-R, R]$ when $j \geq j_{R}$. The limit set may be the empty set. Let us assume that $M_{j}$ satisfy (4.7) uniformly in $j$ which means that $R(\varepsilon)$ does not depend on $j$. If these $M_{j}$ converge to a limit set $M$, then $d \leq D_{-}(M)$. We now return to the proof of Lemma 4.1 and denote by $d$ the lower density of $M \subset \mathbb{Z}$. We now embed the vector space of all measures supported by $I$ into the larger space $W_{I}$ consisting of all pseudo-measures supported by $I$. The topology of $W_{I}$ is the weak-star topology defined by the duality between $l^{\infty}$ and $l^{1}$. Then from any sequence $n_{j}, j \in \mathbb{N}$, one can extract a subsequence $n_{j}^{\prime}$ such that the sequence of sets $M_{j}=M-n_{j}^{\prime}$ weakly converges to a limit set $M^{\prime}$. The lower density of this set $M^{\prime}$ is still larger than or equal to $d$. We then argue by contradiction. If (4.8) does not hold, one can find a sequence $\mu_{j}$ of measures carried by $I$ and a sequence $n_{j}$ of integers such that $\left|\hat{\mu}_{j}\left(n_{j}\right)\right| \geq 1-1 / j$ and $\left\|\hat{\mu}_{j}\right\|_{l^{\infty}(\mathbb{Z})}=1$ while $\left\|\hat{\mu}_{j}\right\|_{l^{\infty}(M)} \leq 1 / j$. Multiplying $\mu_{j}$ by a suitable constant $c$ of modulus 1 we can assume $\hat{\mu}_{j}\left(n_{j}\right) \geq 1-1 / j$. Let $\hat{\nu}_{j}(x)=\hat{\mu}_{j}\left(x+n_{j}\right)$ and let $\nu$ be a weak limit of a subsequence of these $\nu_{j}$. Then $\nu$ is a pseudo-measure supported by $I, \hat{\nu}=0$ on $M^{\prime}$ while $\hat{\nu}(0)=1$ and $\hat{\nu} \in l^{\infty}$. This contradicts the classical results on the density of zeros of entire functions of exponential type. The reader may consult "Gap and density theorems" by Norman Levinson, Chapter III, Theorem VIII. Here the entire function is the Fourier-Stieljes transform $F(z)=\int \exp (-2 \pi z x) d \nu(x)$ of $\nu$. The same proof yields the second statement in Lemma 4.1.

We now complete the proof of Proposition 4.1. Once again we use the fact that $\Gamma$ is dense in $\mathbb{T}^{2}$ and we have

$$
\sup _{k \in \mathbb{Z}}|\hat{\mu}(-k)|=\sup _{k \in \mathbb{Z}}|F(k \sqrt{2}, k \sqrt{3})|=\|F\|_{\infty} .
$$

Then (4.6), (4.8), and (4.9) yield the required estimate (4.3). 
For studying the dependence in $\Omega$ of the constant $C_{\Omega, I}$, we introduce a new definition

Definition 4.2. Let $W$ be a sequence of positive numbers $w_{j}, j \in \mathbb{N}$. We say that an open set $\Omega \subset \mathbb{T}^{2}$ is $W$-thick if one can find a sequence of pairwise disjoint discs $Q_{j} \subset \Omega$ s.t. $\left|Q_{j}\right|>w_{j}>0, j \in \mathbb{N}$.

For a given $\Omega$ one can always find a sequence $W$ such that $\Omega$ is $W$-thick. When $W$ is given, if an open set $\Omega$ is $W$-thick, then $\Omega$ contains " $W$-large discs".

Proposition 4.2. We have $C=C_{\Omega, I} \leq C_{W, I}$ in Proposition 4.1 if there exists a sequence $W=\left(w_{j}\right)_{j \in \mathbb{N}}$ with the following properties:

(a) $\sum_{0}^{\infty} w_{j}>|I|$

(b) $\Omega$ is W-thick.

We now check Proposition 4.2. We set $E=Q_{1} \cup \ldots \cup Q_{N}$ where $w_{1}+$ $\ldots+w_{N}>|I|$. Now $N$ is fixed as everything else but the centers of the discs $Q_{j}$. The arguments used in Proposition 4.1 apply here with $E$ replacing $\Omega$ and the proof of Proposition 4.2 ends with the following lemma

Lemma 4.2. If $r>0$ is given, $x \in \mathbb{T}^{2}$ is arbitrary, then the set of integers defined by $M=\{k \in \mathbb{Z}, \gamma(k) \in B(x, r)\}$ has a uniform density given by $\pi r^{2}$. Moreover the estimates (2.16) are uniform in $x$.

We turn to $L^{2}$ estimates.

Proposition 4.3. We still assume that $\Omega \subset \mathbb{T}^{2}$ is an open set whose measure satisfies $|\Omega|>|I|$. Then there exists a constant $C=C(\Omega, I)$ such that for any continuous function $F$ on $\mathbb{T}^{2}$ whose spectrum is included in $\Lambda_{I}$, one has

$$
\|F\|_{2} \leq C\|F\|_{L^{2}(\Omega)}
$$

Moreover $C(\Omega, I) \leq C(W, I)$ if there exists a sequence $W=\left(w_{j}\right)_{j \in \mathbb{N}}$ with the following properties: (a) $\sum_{0}^{\infty} w_{j}>|I|$ and (b) $\Omega$ is $W$-thick.

Proposition 2.2 and Proposition 4.2 imply Proposition 4.3. We now state our main theorem which contains Theorem 2.1.

Theorem 4.1. With the preceding notations, let $K \subset \mathbb{Z}^{2}$ be a compact set such that $|K|<|I|$. Then $\Lambda_{I}$ is a set of stable sampling for $Y_{K}^{p}$ for $1 \leq p \leq$ $\infty$. In other words for any sequence $f \in l^{p}\left(\mathbb{Z}^{2}\right)$ of Fourier coefficients of a function $F$ is supported by $K$, one has

$$
\left(\sum_{k \in \mathbb{Z}^{2}}|f(k)|^{p}\right)^{1 / p} \leq C\left(\sum_{\lambda \in \Lambda_{I}}|f(\lambda)|^{p}\right)^{1 / p} .
$$

Moreover $C=C_{K, I} \leq C_{W, I}$ when the complement $\Omega$ of $K$ satisfies:(a) $\sum_{0}^{\infty} w_{j}>1-|I|$ and $(b) \Omega$ is $W$-thick. 
For proving Theorem 4.1 we denote by $J=I^{c}$ the complement of $I$ in $\mathbb{T}$. We observe that $J \subset \mathbb{T}$ is still an arc. It suffices now to observe that the complement of $\Lambda_{I}$ in $\mathbb{Z}^{2}$ is $M=\Lambda_{J}$ and to apply Proposition 3.1. The last assertion in Theorem 4.1 is following from the corresponding statement in Lemma 4.1. One cannot hope for a uniform estimate where $C=C\left(a_{1}, a_{2}\right)$ would only depend on the positive numbers $a_{1}=|K|$ and $a_{2}=|I|$. A counterexample will be given in Section 4.

We are ready to prove Theorem 2.4. The organization of the proof is the same as for Theorem 4.1. Once more Proposition 4.1 is being used together with Theorem 2.3. As it was said before, we use the fact that the indicator function of $\Lambda_{I}$ is a multiplier for for $\mathcal{F} L^{p}$ when $1<p<\infty$. This comes from the transference arguments developed by Coifman and Weiss in [2].

\section{Completion of the proof of Theorem 1.1}

As above $\gamma: \mathbb{Z} \mapsto \mathbb{T}^{2}$ is defined by $\gamma(k)=\Pi(k \sqrt{2}, k \sqrt{3})$. We denote by $\Gamma=\gamma(\mathbb{Z})$ the range of $\gamma$. The proof of assertion (b) of Theorem 1.1 relies on the following theorem:

Theorem 5.1. Let $K \subset \mathbb{T}^{2}$ be a compact set such that $|K|<\alpha$. If $x_{0}$ belongs to $\Gamma \backslash K$, there exists an atomic measure $\sigma$ on $\mathbb{T}^{2}$ enjoying the following three properties

$$
\begin{gathered}
\sigma \geq 0 \text { and } \sigma\left(\left\{x_{0}\right\}\right)=1 \\
\sigma(K)=0
\end{gathered}
$$

the Fourier transform $\hat{\sigma}$ of $\sigma$ is supported by $\Lambda_{\alpha}$.

A bound on the total mass $\|\sigma\|$ of $\sigma$ will be given below. We postpone the proof of Theorem 5.1 and prove (c) in Theorem 1.1. Let $K$ be the closed support of $F$. We know that $F \geq 0$ and $|K|<\alpha$. We want to compare $F$ to a competitor $u$ which verifies $u \geq 0$ and $\hat{u}=\hat{F}$ on $\Lambda_{\alpha}$. Our first claim is that the proof reduces to the case where $F$ and $u$ are continuous functions. For proving this remark let us consider $\varphi_{j}(x)=j^{2} \varphi(j x)$ where $\varphi \in \mathcal{C}_{0}^{\infty}, \varphi \geq 0$ and $\int \varphi(x) d x=1$. Replacing $F$ and $u$ by $F_{j}=F * \varphi_{j}$ and $u_{j}=u * \varphi_{j}$, we have $\hat{F}_{j}(k)=\hat{u}_{j}(k), k \in \Lambda_{\alpha}$. Moreover the support of $F_{j}$ is contained in $K_{j}=\left\{x ; \operatorname{dist}(x, K) \leq \frac{C}{j}\right\}$ where $C$ depends on the support of $\varphi$. We have $\lim \left|K_{j}\right|=|K|<\alpha$ which implies $\left|K_{j}\right|<\alpha$ for $j \geq j_{1}$. If we can prove that $F_{j}=u_{j}$ for $j \geq j_{0}$ then we can conclude. We now restrict our attention to $F_{j}, u_{j}$ and $K_{j}$. We forget the subscript $j$ and assume that $F$ and $u$ are smooth. Replacing $K$ by a slightly larger set we can assume that $K$ is Riemann integrable. 
We have $\Lambda_{\alpha}=-\Lambda_{\alpha}$. Then if $x_{0} \notin K$ we use Theorem 5.1 and write

$$
\begin{aligned}
0 & \leq \int_{\mathbb{T}^{2}} u d \sigma=\sum_{k \in \mathbb{Z}^{2}} \hat{u}(k) \hat{\sigma}(-k)=\sum_{k \in \Lambda_{\alpha}} \hat{u}(k) \hat{\sigma}(-k) \\
& =\sum_{k \in \Lambda_{\alpha}} \hat{F}(k) \hat{\sigma}(-k)=\int_{\mathbb{T}^{2}} F d \sigma
\end{aligned}
$$

But $F$ vanishes on $\mathbb{T}^{2} \backslash K$ and $\sigma(K)=0$. Therefore $\int_{\mathbb{T}^{2}} F d \sigma=0$ which together with (5.4) implies $\int_{\mathbb{T}^{2}} u d \sigma=0$. Finally we use again the fact that $u$ and $\sigma$ are nonnegative. We have

$$
0 \leq u\left(x_{0}\right)=\sigma\left(\left\{x_{0}\right\}\right) u\left(x_{0}\right) \leq \int_{\mathbb{T}^{2}} u d \sigma=0
$$

and $u\left(x_{0}\right)=0$. Since the subgroup $\Gamma=\{\Pi(k \sqrt{2}, k \sqrt{3}), k \in \mathbb{Z}\}$ is dense in $\mathbb{T}^{2}$, we obtain $u=0$ on $\mathbb{T}^{2} \backslash K$. Therefore $u$ is supported by $K$ and Theorem 2.1 yields the required result.

The proof of (b) in Theorem 1.1 is almost trivial. Indeed let us assume that a competitor $u$ exists with $\|u\|_{1} \leq\|F\|_{1}$. We decompose $u$ into a sum $u=u_{1}-u_{2}+i u_{3}$ where $u_{1}$ and $u_{2}$ are nonnegative functions or measures with disjoint supports and $u_{3}$ is real valued. Since $0 \in \Lambda_{\alpha}$ and $F$ is nonnegative we have

$$
\begin{aligned}
\hat{u}_{1}(0)-\hat{u}_{2}(0)+i \hat{u}_{3}(0) & =\hat{u}(0)=\hat{F}(0)=\|F\|_{1} \geq\|u\|_{1} \\
& \geq\left\|u_{1}-u_{2}\right\|_{1}=\hat{u}_{1}(0)+\hat{u}_{2}(0) .
\end{aligned}
$$

Therefore $\hat{u}_{3}(0)=\hat{u}_{2}(0)=0$ which implies $u_{2}=0$ since $u_{2}$ is nonnegative. Finally the first and the last term in (5.6) are equal. Therefore all terms in (5.6) are equal and $\|u\|_{1}=\|F\|_{1}$. Then (5.6) reduces to $\left\|u_{1}\right\|_{1}=\hat{u}_{1}(0)=$ $\hat{u}(0)=\|F\|_{1}=\|u\|_{1}$ which implies $u_{3}=0$. Finally $u$ is nonnegative and it now suffices to use (c) in Theorem 1.1.

We now prove Theorem 5.1. Let $S=\{k \in \mathbb{Z} ; \gamma(k) \in K\}$. Since $K$ is Riemann integrable $S$ has a uniform density $d$ which is given by $d=|K|$. We forget $\mathbb{T}^{2}$ and focus on $\mathbb{T}$ and $\mathbb{Z}$. The Fourier coefficients of a function $F \in L^{1}(\mathbb{T})$ are defined by $c(k)=\int_{\mathbb{T}} F(x) \exp (-2 \pi i k x) d x$. The proof relies on the following lemmas.

Lemma 5.1. Let us assume that $S \subset \mathbb{Z}$ has a uniform density $d \in(0,1)$. Let $J \subset \mathbb{T}$ be an interval centered at 0 with length $|J|>d$. Then there exists a constant $C$ such that if $k_{0} \notin S$ there exists a function $h \in L^{2}(J)$ such that $\hat{h}(k)=0$ for every $k \in S, \hat{h}\left(k_{0}\right)=1$ and $\|h\|_{L^{2}(J)} \leq C$. 
We then have

Lemma 5.2. Let us assume that $S \subset \mathbb{Z}$ has a uniform density $d \in(0,1 / 2)$. Let $I \subset \mathbb{T}$ be an interval centered at 0 with length $|I|>2 d$. Then there exists a constant $C$ such that if $k_{0} \notin S$ there exists a continuous function $\phi$ supported by $I$ such that $\hat{\phi}(k) \geq 0, k \in \mathbb{Z}, \hat{\phi}(k)=0$ for every $k \in S, \sum_{-\infty}^{+\infty} \hat{\phi}(k) \leq C$, and $\hat{\phi}\left(k_{0}\right)=1$.

The proof of Lemma 5.2 is obvious if Lemma 5.1 is accepted. It suffices to define $\phi$ by $\phi=h * \tilde{h}$ where $\tilde{h}(x)=\bar{h}(-x)$. We now return to Lemma 5.1. The proof is based on the following estimate.

Lemma 5.3. Let us assume that $S \subset \mathbb{Z}$ has a uniform density $d \in(0,1)$. Let $J \subset \mathbb{T}$ be any interval of length larger than $d$. Then there exists a positive constant $\beta$ such that for any $l \notin S$ and for any sequence $c(k) \in l^{2}(S)$, we have

$$
\left\|\exp (2 \pi i l x)-\sum_{k \in S} c(k) \exp (2 \pi i k x)\right\|_{L^{2}(J)} \geq \beta
$$

This estimate implies Lemma 5.1 with $C=1 / \beta$. We now prove Lemma 5.3 using the simplest form of Beurling's theorem [1]. Here is the statement

Theorem 5.2. Let $\Lambda \subset \mathbb{Z}$ and let $D^{+}(\Lambda)=\lim _{R \rightarrow+\infty} R^{-1} \sup _{k \in \mathbb{Z}} \#\{\Lambda \cap$ $[k, k+R]\}$ be the upper density of $\Lambda$. Then if the length $|J|$ of an interval $J \subset \mathbb{T}$ satisfies $|J|>D^{+}(\Lambda)$ there exists a constant $C$ such that for every sequence $c(k), k \in \Lambda$,

$$
\sum_{k \in \Lambda}|c(k)|^{2} \leq C^{2} \int_{J}\left|\sum_{k \in \Lambda} c(k) \exp (2 \pi i k x)\right|^{2} d x .
$$

For proving Lemma 5.3 we mimic the proof of Lemma 4.1 and argue by contradiction. Let us denote by $H$ the Hilbert space $L^{2}(J)$. Let us assume that one can find a sequence $l_{j} \notin S$ and some coefficients $c(k, j)$ such that

$$
\left\|\exp \left(2 \pi i l_{j} x\right)-\sum_{k \in S} c(k, j) \exp (2 \pi i k x)\right\|_{H} \leq 1 / j .
$$

The triangle inequality gives

$$
\left\|\sum_{k \in S} c(k, j) \exp (2 \pi i k x)\right\|_{H} \leq 2
$$

Then Beurling's theorem yields

$$
\left(\sum_{k \in S}|c(k, j)|^{2}\right)^{1 / 2} \leq 2 C
$$


This being said, we rewrite (5.9) as

$$
\left\|1-\sum_{k \in S_{j}} c\left(k+l_{j}, j\right) \exp (2 \pi i k x)\right\|_{H} \leq 1 / j .
$$

with $S_{j}=S-l_{j}$.

We now use the fundamental assumption that $S$ has a uniform density. Therefore we can replace the sequence $S_{j}$ by a subsequence such that $S_{j} \rightarrow$ $S^{\prime}$. It means that for each $R \geq 1$ and $j \geq j(R)$ we have $S_{j} \cap[-R, R]=S^{\prime} \cap$ $[-R, R]$. The density of $S^{\prime}$ is still $d$ and is uniform. Similarly we set $c_{j}(k)=$ $c\left(k+l_{j}, j\right)$ and we can also assume that $c_{j}(k)$ weakly converges to $c^{\prime}(k), k \in$ $\mathbb{Z}$. These two convergences imply the weak convergence $F_{j}(x)=\sum_{k \in S_{j}} c(k+$ $\left.l_{j}, j\right) \exp (2 \pi i k x) \rightarrow F(x)=\sum_{k \in S^{\prime}} c^{\prime}(k) \exp (2 i k x)$ as $j$ tends to infinity. This weak convergence refers to the weak topology in the Hilbert space $H$. But $0 \notin S^{\prime}$ since $0 \notin S_{j}$. Finally (5.12) yields $1=\sum_{k \in S^{\prime}} c^{\prime}(k) \exp (i k x)$ in $L^{2}(J)$ which contradicts Beurling's theorem applied to $S^{\prime} \cup\{0\}$.

We now return to Theorem 5.1. We have $|K|<\alpha$. This compact set $K$ is replaced by a slightly larger compact set $L$ which is Riemann integrable of measure $|L|<\alpha$. Then the set $S=\{k \in \mathbb{Z} ; \gamma(k) \in L\}$ has a uniform density $d=|L|<\alpha$. Lemma 5.2 is applied to $I=[-\alpha, \alpha]$ when $k_{0}$ is defined by $\gamma\left(k_{0}\right)=x_{0}$. The atomic measure $\sigma$ is defined by

$$
\sigma=\sum_{-\infty}^{\infty} \hat{\phi}(k) \delta_{\gamma(k)}
$$

where $\delta_{a}$ is the Dirac mass at $a$. Then $\sigma$ is nonnegative. We have $\hat{\phi}(k)=0$ whenever $\gamma(k) \in L$. This implies $\sigma(L)=0$. We also have $\sigma \geq \delta_{x_{0}}$ since $\hat{\phi}\left(k_{0}\right)=1$. Finally

$$
\begin{aligned}
\hat{\sigma}(-p,-q) & =\sum_{-\infty}^{\infty} \hat{\phi}(k) \exp [2 \pi i(p \sqrt{2}+q \sqrt{3}) k] \\
& =\phi(p \sqrt{2}+q \sqrt{3})=0 \text { when }(p, q) \notin \Lambda_{\alpha} .
\end{aligned}
$$

This concludes the proof of Theorem 5.1.

We now prove Theorem 1.2. This proof relies on an estimate of the total mass of the measure $\sigma$ in Theorem 5.1. This estimate will depend on the growth of the function $\beta(\varepsilon)$ of $\varepsilon>0$ defined by $\beta(\varepsilon)=|K+B(0, \varepsilon)|$. We begin with a few remarks.

Lemma 5.4. For every positive number $\eta$ there are finitely many Riemann integrable compact sets $L \in \mathcal{L}$ such that for every compact set $K \subset \mathbb{T}^{2}$ one can find $L \in \mathcal{L}$ such that $K \subset L \subset K+B(0, \eta)$. 
The proof of Lemma 5.4 is trivial. One uses a "fine grid" on $\mathbb{T}^{2}$ with step size $\eta / 2$ and $\mathcal{L}$ is simply the collection of all finite unions of squares delimited by this grid.

Proposition 5.1. With the preceding notations, we let $\eta$ be small enough so that the measure $\beta(2 \eta)$ of $K+B(0,2 \eta)$ is less than $\alpha$. We also assume that $\eta$ is smaller than the distance from $x_{0}$ to $K$. Then in Theorem 5.1 the total mass of $\sigma$ does not exceed $C(\alpha, \beta(\eta))$.

The value of $\eta$ depends on the geometrical structure of the compact set $K$ and not only on the measure of $K$. The proof of Proposition 5.1 is not difficult. We first use Lemma 5.4 and enlarge $K$ into $L \in \mathcal{L}$ with $K \subset L \subset$ $K+B(0, \eta)$ where $\eta$ is small enough to ensure $\beta(2 \eta)=|K+B(0,2 \eta)|<\alpha$. If $\eta$ is small enough we also have $x_{0} \notin L$. Finally it suffices to rewrite the proof of Theorem 5.1 and to keep track of the constants which come out. As $\eta$ tends to 0 , the cardinality of $\mathcal{L}$ blows up and so does the mass of $\sigma$.

We now return to the proof of Theorem 1.2. As we did in the proof of Theorem 1.1, we can assume that $u$ and $F$ are continuous. Let $K$ be the closed support of $F$. We define $L$ by Lemma 5.4 and let $x_{0} \notin L$. The total mass of the measure $\sigma$ provided by Theorem 3.1 does not exceed $C(\alpha, \beta)$ which is defined by Proposition 5.1. Keeping notations as simple as possible, we write $\Lambda$ for $\Lambda_{\alpha}$.

Then $u \geq 0$ implies:

$$
\begin{aligned}
0 \leq u\left(x_{0}\right) & \leq \int u d \sigma=\sum_{\Lambda} \hat{u}(\lambda) \hat{\sigma}(-\lambda)=\sum_{\Lambda} \hat{f}(\lambda) \hat{\sigma}(-\lambda)+\sum_{\Lambda} \hat{R}(\lambda) \hat{\sigma}(-\lambda) \\
& =\int f d \sigma+\int R d \sigma=I_{1}+I_{2} .
\end{aligned}
$$

Then $I_{1}=0$ since $F$ is supported by $L$ and $\sigma(L)=0$. Moreover $\|R\|_{\infty} \leq \varepsilon$ and $\|\sigma\| \leq C$ imply $\left|I_{2}\right| \leq C \varepsilon$. We obtain $0 \leq u\left(x_{0}\right) \leq C^{\prime} \varepsilon$. This estimate is uniform in $x_{0} \notin L$. We now write $u=u_{1}+u_{2}$ where $u_{2}$ is the product between $u$ and the indicator function of $L$. We then have $\left\|u_{1}\right\|_{\infty} \leq C^{\prime} \varepsilon$. This implies $\hat{u}_{2}(\lambda)=\hat{f}(\lambda)+\hat{r}_{2}(\lambda), \lambda \in \Lambda$, where $\left\|r_{2}\right\|_{\infty} \leq C^{\prime \prime} \varepsilon$. Theorem 1.2 results from the following lemma applied to $u=u_{2}-f$ and $v=r_{2}$

Lemma 5.5. If $K$ is a compact set of measure $|K|<2 \alpha$, then there exists a constant $C$ such that for any function $u$ supported by $K$ we have

$$
\|u\|_{\infty} \leq C_{K} \inf \left\{\|v\|_{\infty} ; \hat{u}(\lambda)=\hat{v}(\lambda), \lambda \in \Lambda_{\alpha}\right\}
$$

where the infimum runs over all competitors $v$ without any restriction on their supports. In other words $\Lambda_{\alpha}$ is a set of stable sampling for $E_{K}^{\infty}$. 
By duality Lemma 5.5 implies the following. Every measure $\mu$ on $K$ is the restriction to $K$ of a measure $\nu$ whose Fourier expansion is given by $\nu=\sum_{k \in \Lambda_{\alpha}} a(k) \exp (2 \pi k \cdot x)$. This is almost Theorem 5.1 when $K$ is replaced by $K \cup\left\{x_{0}\right\}$. But Theorem 5.1 says more since it says that that $\nu$ is nonnegative. That explains why another proof was used for proving Theorem 5.1. Let us observe that in Theorem 5.1 the measure $|K|$ is less than $\alpha$ while here it suffices to assume $|K|<2 \alpha$.

We now prove Lemma 5.5. We write $\Lambda$ for $\Lambda_{\alpha}$ and we know that $\hat{u} \mathbf{1}_{\Lambda}=$ $\hat{v} \mathbf{1}_{\Lambda}$. As we did in proving Theorem 2.3, we split $u$ into a sum $u_{1}+u_{2}$ where $\hat{u}_{1}=\hat{u} \mathbf{1}_{\Lambda}$. This crude definition of $u_{1}$ will be modified at the end of the proof. Let us denote by $M$ the complement of $\Lambda$ in $\mathbb{Z}^{2}$ and by $\Omega$ the complement of $K$ in $\mathbb{T}^{2}$. Then $\hat{u}_{2}$ is supported by $M$. The measure of $\Omega$ exceeds the density of $M$ and Proposition 4.1 yields $\left\|u_{2}\right\|_{\infty} \leq C\left\|u_{2}\right\|_{L^{\infty}(\Omega)}$. But $u_{1}+u_{2}=0$ on $\Omega$ which implies $\left\|u_{2}\right\|_{\infty} \leq C\left\|u_{1}\right\|_{L^{\infty}(\Omega)} \leq C\left\|u_{1}\right\|_{\infty}$. The proof would finish if we could believe that $\hat{u}_{1}=\hat{v} \mathbf{1}_{\Lambda}$ implies $\left\|u_{1}\right\|_{\infty} \leq C\|v\|_{\infty}$. This cannot be true since the indicator function of $\Lambda$ is not the Fourier-Stieljes transform of a measure on $\mathbb{T}^{2}$. For facing this issue we introduce a function $\beta$ on $\mathbb{T}$ which is 1 on $[-\alpha+\varepsilon, \alpha-\varepsilon]$, is smooth and is supported by $[-\alpha, \alpha]$. We define $B(p, q)=\beta(p \sqrt{2}+q \sqrt{3}),(p, q) \in \mathbb{Z}^{2}$, on $\mathbb{Z}^{2}$. Then $B(p, q),(p, q) \in \mathbb{Z}^{2}$, are the Fourier coefficients of an atomic measure $\nu$. Finally we define $u_{1}$ by $\hat{u}_{1}(p, q)=B(p, q) \hat{u}(p, q)$ and proceed as above. The support of $u_{2}=u-u_{1}$ is contained in $M^{\prime}$ which is defined as the set of all pairs $(p, q) \in \mathbb{Z}^{2}$ such that there exists a $r \in \mathbb{Z}$ with $|p \sqrt{2}+q \sqrt{3}-r|>\alpha-\varepsilon$. Therefore the density of $M^{\prime}$ is given by $1-2 \alpha+2 \varepsilon$ which is smaller than the measure of $\Omega$ when $\varepsilon$ is sufficiently small. This being said, the argument used in the "wrong proof" is valid and yields Lemma 5.5.

\section{Counterexamples}

The following counterexamples show that Theorem 1.1 is sharp.

Lemma 6.1. There exist two nonnegative continuous functions $u$ and $v$ on $\mathbb{T}^{2}$ such that $u \neq v$ while $\hat{u}(k)=\hat{v}(k), k \in \Lambda_{\alpha}$.

This lemma says that we cannot have uniqueness in (c) of Theorem 1.1 if the information concerning the measure of the support of $F$ is dropped. The proof is simple. Let $\theta(t)$ be the triangle function on $\mathbb{T}=\mathbb{R} / \mathbb{Z}$ defined by $\theta(1 / 2)=1, \theta(\alpha)=\theta(-\alpha)=0, \theta$ being affine on $[\alpha, 1 / 2]$ and on $[1 / 2,1-\alpha]$. Then

$$
\theta(t)=\sum_{-\infty}^{\infty}(-1)^{k} \tau_{k} \exp (2 \pi i k t),
$$

where $\tau_{k}>0$. 
We now consider the atomic measure $\tau=\sum_{-\infty}^{\infty}(-1)^{k} \tau_{k} \delta_{\gamma(k)}$ and we have, as above, $\hat{\tau}(p, q)=0$ on $\Lambda_{\alpha}$. The atomic measure $\tau$ can be written as the difference $\sigma-\rho$ where $\sigma=\sum_{-\infty}^{\infty} \tau_{2 k} \delta_{\gamma(2 k)}$. Then we have $\sigma>0, \rho>0$. To prove Lemma 6.1 it suffices to use the same approximation to the identity as in Section 5 and to define $u_{j}$ and $v_{j}$ by $u_{j}=\sigma * \varphi_{j}, v_{j}=\rho * \varphi_{j}$ where $\varphi \geq 0$. We have $\hat{u}_{j}=\hat{v}_{j}$ on $\Lambda_{\alpha}$ but $u_{j} \neq v_{j}$ if $j$ is large enough.

In the same spirit we have

Lemma 6.2. For every positive $\varepsilon$ there exist a compact set $K \subset \mathbb{T}^{2}$ of measure not exceeding $\varepsilon$ and a continuous function $F$ supported by $K$ such that $F$ is not the argument of the problem

$$
\inf \left\{\|u\|_{1} ; \hat{u}(\lambda)=\hat{F}(\lambda), \lambda \in \Lambda_{\alpha}\right\} .
$$

This lemma says that $F \geq 0$ is playing a key role in (b) of Theorem 1.1. We use the same atomic measure $\tau$ as before and split it into $\tau_{N}+\rho_{N}$ where $\tau_{N}=\sum_{|k| \leq N}(-1)^{k} \tau_{k} \delta_{\gamma(k)}$. Next we set $F_{N}=\tau_{N} * \phi_{N}, r_{N}=-\rho_{N} * \phi_{N}$ where $\phi_{N}(x)=N^{2} \phi(N x)$. The function $F$ we are looking for is $F_{N}$. We let $K=K_{N}$ be the closed support of $F_{N}$. Then the measure of $K_{N}$ does not exceed $2 / N$. Moreover

$$
\hat{F}_{N}(\lambda)=\hat{r}_{N}(\lambda), \lambda \in \Lambda_{\alpha}
$$

Finally we have $\left\|F_{N}\right\|_{1}>\left\|r_{N}\right\|_{1}$ when $N$ is large enough since the latter tends to 0 as $N$ tends to infinity while $\left\|F_{N}\right\|_{1} \geq c>0$. To prove this last remark, we write $F_{N}=\tau * \phi_{N}-r_{N}$ and the triangle inequality yields $\left\|F_{N}\right\|_{1} \geq\left\|\tau * \phi_{N}\right\|_{1}-\left\|r_{N}\right\|_{1}$. It now suffices to show that $\left\|\tau * \phi_{N}\right\|_{1} \geq c>0$. Arguing by contradiction we would obtain $\left\|\tau * \phi_{N_{j}}\right\|_{1} \rightarrow 0$ as $j$ tends to infinity. This would imply $\tau=0$. Therefore the challenger $r_{N}$ is winning against $F_{N}$. This ends the proof of Lemma 6.2.

The requirement $\alpha>\beta$ in Theorem 1.1 is sharp. It cannot be replaced by $\alpha<\beta$ as our next lemma shows. However this does not settle the case $\alpha=\beta$ in Theorem 1.1.

Lemma 6.3. If $0<\alpha<\alpha_{1}<1 / 2$, there exist two nonnegative functions $F$ and $G$ such that:

- $\hat{F}(k)=\hat{G}(k), k \in \Lambda_{\alpha}$

- the measure of the closed support $K$ of $F$ does not exceed $\alpha_{1}$

- $F \neq G$. 
We start with the rectangle $K \subset \mathbb{T}^{2}$ defined by $-1 / 2 \leq x_{1} \leq 1 / 2$ and $-\alpha_{1} \leq x_{2} \leq \alpha_{1}$. We let $Z \subset \mathbb{Z}$ be the set of all $k \in \mathbb{Z}$ such that $\gamma(k) \notin K$. Then $Z=-Z$ and the uniform density of $Z$ is $1-2 \alpha_{1}<1-2 \alpha$. If $k_{0} \notin Z$, Lemma 5.1 yields a function $\theta \in L^{2}([\alpha, 1-\alpha])$ which is supported by $[\alpha, 1-\alpha]$ and such that $\hat{\theta}(k)=0$ if $k \in Z$ and $\hat{\theta}\left(k_{0}\right)=1$. We then replace $\theta$ by $\psi(t)=\theta(t)+\bar{\theta}(-t)$ in such a way that $\hat{\theta}(k)$ is replaced by $\hat{\psi}(k)=2 \Re \hat{\theta}(k)$. The interval $[\alpha, 1-\alpha]$ viewed as an arc in $\mathbb{T}$ is symmetric with respect to 0 . Therefore this function $\psi$ is still supported by $[\alpha, 1-\alpha]$. Moreover $\hat{\psi}\left(k_{0}\right)=2$ and $\hat{\psi}$ is real valued. By regularization $\psi$ can be assumed to belong to $\mathcal{C}_{0}^{\infty}([\alpha, 1-\alpha])$. Indeed the above construction can be applied to the interval $\left[\alpha^{\prime}, 1-\alpha^{\prime}\right]$ where $\alpha<\alpha^{\prime}<\alpha_{1}$. This is letting enough room for a convolution with a smooth approximation of the identity. We now consider the atomic measure

$$
\tau=\sum_{-\infty}^{\infty} \hat{\psi}(k) \delta_{\gamma(k)} .
$$

Then $\tau$ is supported by $K$. Indeed $\hat{\psi}(k)=0$ if $\gamma(k) \notin K$. By construction $\hat{\tau}(p, q)=0$ when $(p, q) \in \Lambda_{\alpha}$. We then define $T_{j} \in \mathcal{C}^{\infty}\left(\mathbb{T}^{2}\right)$ by $T_{j}=\tau * \varphi_{j}$ where $\varphi_{j}$ is defined as above. Then $T_{j}$ is supported by $K_{j}=\left\{-1 / 2 \leq x_{1} \leq\right.$ $\left.1 / 2 ;-\alpha_{1}-1 / j \leq x_{2} \leq \alpha_{1}+1 / j\right\}$. Let $A_{j}=\left\{x \in K_{j} ; T_{j}(x)>0\right\}$ and $B_{j}=\left\{x \in K_{j} ; T_{j}(x) \leq 0\right\}$. Since $A_{j}$ and $B_{j}$ are partitioning $K_{j}$ we either have $\left|A_{j}\right| \leq \alpha_{1}+1 / j$ or $\left|B_{j}\right| \leq \alpha_{1}+1 / j$. Let $F_{j}=T_{j} \mathbf{1}_{A_{j}}, G_{j}=-T_{j} \mathbf{1}_{B_{j}}$. Then $T_{j}=F_{j}-G_{j}$ and $\hat{F}_{j}=\hat{G}_{j}$ on $\Lambda_{\alpha}$ by construction. Moreover $F_{j} \geq 0$ and $G_{j} \geq 0$. One of the two functions $F_{j}$ or $G_{j}$ is the counterexample. We cannot have $F_{j}=G_{j}$ for infinitely many $j^{\prime}$ s since it would imply $T_{j}=0$ for infinitely many $j^{\prime}$ s and $\tau=0$.

We now turn to the issue discussed in Theorem 4.1. We wanted to know if the contant $C$ in (4.11) depends only on $|I|-|K|$. A counterexample is given by the following theorem where $\Lambda=\Lambda_{\alpha}$. This theorem shows that the geometry of $K$ enters in $C$.

Theorem 6.1. For every $\eta>0$ and every integer $N$ there exist a compact set $K \subset \mathbb{T}^{2}$ whose measure does not exceed $\eta$ and a function $F \in L^{2}\left(\mathbb{T}^{2}\right)$ which is supported by $K$ and fulfils the following two conditions $\|F\|_{2}=1$ while

$$
\sum_{\lambda \in \Lambda}|\hat{F}(\lambda)|^{2} \leq N^{-2}
$$

Let $M$ be the complement of $\Lambda$ in $\mathbb{Z}^{2}$. The proof of Theorem 6.1 begins with the following lemma 
Lemma 6.4. Keeping the same notations as above, there exist a compact set $K$ of measure not exceeding $\eta$ and a function $g$ such that

- the Fourier transform of $g$ is supported by $M$

- $\|g\|_{2}=1$

- $\int_{K^{c}}|g|^{2} d x \leq N^{-2}$.

Here $K^{c}=\mathbb{T}^{2} \backslash K$. We first accept this lemma and prove Theorem 6.1. We let $F$ be the product between $g$ and the indicator function of $K$. Then $\|F-g\|_{2} \leq N^{-1}$ which implies $\sum_{\lambda \in \Lambda}|\hat{F}(\lambda)-\hat{g}(\lambda)|^{2} \leq N^{-2}$. But $\hat{g}(\lambda)=0$ if $\lambda \in \Lambda$. Therefore $F$ is enjoying the properties listed in Theorem 6.1.

We now prove Lemma 6.4. Let $\theta$ and the atomic measure $\tau$ be defined as in Lemma 6.1. We consider the atomic measure $\tau=\sum_{-\infty}^{\infty}(-1)^{k} \tau_{k} \delta_{\gamma(k)}$ and we have, as above, $\hat{\tau}(p, q)=0$ on $\Lambda$. As we did in the proof of Lemma 6.2, we split $\tau$ into $\tau_{N}+\rho_{N}$. We now consider $g=g_{\varepsilon}=\tau * \phi_{\varepsilon}=u_{N, \varepsilon}+v_{N, \varepsilon}$ where $u_{N, \varepsilon}=\tau_{N} * \phi_{\varepsilon}$ and $v_{N, \varepsilon}=\rho_{N} * \phi_{\varepsilon}$ with $\phi_{\varepsilon}(x)=\varepsilon^{-1} \phi(x / \varepsilon), \phi$ being supported by $|x| \leq 1$ and normalized in $L^{2}$. Then $u_{N, \varepsilon}$ is supported by the union $K_{N, \varepsilon}$ of $2 N+1$ discs of measure $\pi \varepsilon^{2}$. Therefore the measure of $K$ does not exceed $\pi(2 N+1) \varepsilon^{2}$. We now write $u=u_{N, \varepsilon}, v=v_{N, \varepsilon}$ and $K=K_{N, \varepsilon}$ for easing notations. We then observe that the total mass of $\rho_{N}$ is less than $C / N$. It implies $\|v\|_{2} \leq C / N$ uniformly on $\varepsilon$. Once $N$ is fixed, $\varepsilon$ can be chosen small enough so that the supports of the $2 N+1$ terms in the expansion of $u$ have disjoint supports. Then $C \leq\|u\|_{2} \leq C^{\prime}$ where $C$ and $C^{\prime}$ are two positive constants. The triangle inequality implies the same conclusion for $g$. Finally the norm in $L^{2}\left(K^{c}\right)$ of $g$ coincides with that of $v$ since $u$ is supported by $K$. But $\|v\|_{2} \leq C / N$ which ends the proof.

The following issues will be discussed in some forthcoming work. To our opinion the most exciting problem is to invert the mapping $\Phi: M_{\beta} \mapsto \ell^{2}\left(\Lambda_{\alpha}\right)$ through an efficient algorithm. Theorem 1.1 gives only a partial answer. The second problem is the generalization of our work to the case where images are not delimited by the unit square. A third problem consists in understanding what is happening in the limiting case where the measure of $K$ equals the density of $\Lambda$. The authors are greatly indebted to the anonymous referee. His supportive criticism was a valuable help.

\section{References}

[1] Beurling, A.: Interpolation for an interval on $\mathbb{R}$. In The Collected works of A. Beurling, Vol. 2, 351-365. Lennart Carleson Ed., Birkhäuser, 1989.

[2] Coifman, R. and Weiss, G.: Transference methods in analysis. Conference Board of the Mathematical Sciences Regional Conference Series in Mathematics 31. American Mathematical Society, Providence, R.I., 1976. 
[3] Landau, H. J.: Necessary density conditions for sampling and interpolation of certain entire functions. Acta Math. 117 (1967), 37-52.

[4] Meyer, Y.: Nombres de Pisot, nombres de Salem et analyse harmonique. Lecture Notes in Mathematics 117. Springer-Verlag, Berlin-New York, 1970.

[5] Meyer, Y.: Trois problèmes sur les sommes trigonométriques. Astérisque 1. Société Mathématique de France, Paris, 1973.

[6] Olevskit, A. and Ulanovskit, A.: Universal sampling of band-limited signals. C.R.Math. Acad. Sci. Paris 342 (2006), no. 12, 927-931.

[7] OlevskiI, A. And UlanovskiI, A.: Universal sampling and interpolation of band-limited signals. Geom. Funct. Anal. 18 (2008), no. 3, 1029-1052.

[8] TAO, T.: An uncertainty principle for cyclic groups of prime order. Math. Res. Lett. 12 (2005), no. 1, 121-127.

Recibido: 7 de marzo de 2008

Revisado: 27 de julio de 2008

Basarab Matei

Université Paris Nord

99, Av. Jean-Baptiste Clément

93430 Villetaneuse, France matei@math.univ-paris13.fr

Yves Meyer

CMLA, ENS-Cachan

61, Av. du Président-Wilson

94235 Cachan Cedex, France

yves.meyer@cmla.ens-cachan.fr 